\title{
The Psychophysics of Order and Anisotropy: Comment on Riemer
}

Riemer's paper discusses a neglected yet important topic in the psychological literature: the consequences for psychology (and psychophysics) from the 'anisotropy' of time. The paper presents an argument that there are unique kinds of challenges for psychophysics from such temporal anisotropy:

(a) Challenges because the psychological experience of time has temporal anisotropy and the physical concept of time does not have temporal anisotropy.

(b) Challenges for experimental research which are unique to temporal anisotropy.

Although it is important to consider temporal anisotropy, I think there are reasons to deny the force of both kinds of challenges.

With respect to ' $a$ ', the problem for physics is not so much with temporal anisotropy as it is with temporal order. Although temporal order is a kind of anisotropy, it is significantly different to others with respect to the physics of time. It is global and absolute and irreducible to differences in non-temporal physical structure. Further, the problem of such temporal order is not obviously applicable to psychological phenomena nor, if applicable, particular to it. It is found between fundamental physics and other non-psychological scientific theories (such as thermodynamics).

With respect to ' $b$ ', it is not clear that the problems Riemer raises for experimental research are unique to temporal anisotropy. There are analogous problems with other kinds of anisotropy -- for example, spatial anisotropy.

However, I think that Riemer's reasons are justified if one considers them as coming from intuitive metaphysical positions on time which hold that there is temporal order rather than just temporal anisotropy. Yet, one need not hold these positions on time. And, further, philosophers of time and physicists who accept the physical time assumed in this paper do not accept these positions.

\section{Temporal Anisotropy in Physics and Psychology}

Of the difference between physical and psychological time, Riemer writes:

Physical laws are time-reversal invariant, i.e., they do not imply a specific direction of time $[\ldots]$

$[P]$ sychological time always runs in the same direction, which cannot be reversed or manipulated in psychophysical experiments.

[Riemer, page 2 of review draft]

If these descriptions of physical and psychological time are taken as distinctions between them, then we take those distinctions to be:

a. Psychological time is not time-reversal invariant, implies a specific direction of time, and cannot be reversed in psychophysical experiments. ${ }^{1}$

\footnotetext{
${ }^{1}$ Riemer also refers to manipulation of psychological and physical time. However, as the paper focuses on order, I will ignore all other kinds of manipulation here -- especially as it is not obvious to me that we cannot manipulate other properties of psychological time (it seems we could manipulate psychological duration, for example).
} 
b. Physical time is time-reversal invariant, implies a specific direction of time, and can be reversed in psychophysical experiments.

For psychophysical experiments, the question about the difference rests on the meaning of 'can' in 'a' and 'b'. Can we reverse physical time in psychophysics in a way that we cannot psychological time? I am unclear that we can.

\subsection{Reversibility of Physical and Psychological Time}

In theory, any temporal order of physical events can be reversed and the resulting order of event still be physical. If there is a physical sequence which is event $A$ followed by event $B$, then there is a possible physical sequence which is event $B$ followed event $A$. To use an example from Maudlin (cited in Price 2013, page 285): The motion of an asteroid from Mars to Earth is a physical sequence of events. Reverse the direction of the sequence, and the motion is from Earth to Mars, which is also a physical sequence of events. Although we can distinguish these sequences by their order in time, we do not do so with physical properties. The physical properties of the motion of Mars to Earth are identical to the physical properties of the motion of Earth to Mars.

Further, given Price's 'view from nowhen', the complete physical description of (a) Mars to Earth and (b) Earth to Mars can be different descriptions of one numerically identical set of events. That set of events contains the events of asteroid being at Mars, at Earth, and at various locations in between. The distinction between (a) and (b) is only a distinction between descriptions from different directions in time. If various phenomena are physical when time runs forward, then they are also physical when time runs backward.

As such, we might think that, in theory, we can reverse physical phenomena in time. However, in practice (e.g., in an experimental scenario), can we reverse physical phenomena in time?

In practice, entropy prevents exact inversions of any such sequence. Increasing entropy may be a local phenomenon (Price 1997) but it is also one over which we have little control.

For example, when asking subjects to make judgements, there is a great deal of physical activity involved: presented stimuli cause, in the subject, neural activity and other physiological effects, and a vocal expression of the subject's judgement. All of this activity results in increased disorder (i.e., entropy) in the environment (for example, in the emission of heat).

That I could reverse this sequence is implausible. It would involve making the disordered heat cause an inversion of the subject's verbal reports (they speak backwards), causing neurological and physiological activity, and such subjective activity causing the experimental stimuli (which, so inverted, are then better thought of as experimental effects).

This is not to say that doing this is theoretically impossible; it is just so difficult that it is what one might call practically impossible. Such practical impossibility has consequences for the claim that psychological time is irreversible and physical time is reversible.

Psychological irreversibility may be practically impossible: it may be impossible, for example, to alter the mind such that one forgets what one has just remembered, or to change what is anticipated to what is being remembered, or what is remembered to what has been anticipated. Yet, to meet Riemer's claims, we need to show that there is more to psychological

Commented [SH1]: I don't find this example so helpful. Maybe it could be shortened?

Commented [PSE2R1]: I'm not sure how to shorten it, but happy to try. Could you be more specific about how it is not helpful? 
irreversibility than practical irreversibility. The physical is also practically irreversible. To make the difference between psychological and physical reversibility, we need to show that psychological reversibility is theoretically impossible.

If psychological reversibility is theoretically impossible, then one assumes there is some reason to hold this over and above the practical. It is not obvious that there is such a reason in this paper.

\subsection{Temporal Order}

One reason psychological order might be irreversible in a way physical order is not is because it involves a deeper kind of order than is found in physics. This is what Price calls temporal order. Price denies there is a physical reason for holding there is such order. However, this is not a denial of temporal anisotropy. Temporal order has different commitments to temporal anisotropy.

Riemer writes:

[T] he philosopher Huw Price (1996) claimed that we need to adopt a 'view from nowhen' on physical reality. In order to gain an appropriate description of the physical world, we have to refrain from time-asymmetrical presuppositions about reality. [...] Both directions of physical time are equally probable - and equally unverifiable (Borges, 1936/1999).

However, Price does not deny temporal anisotropy as such. It is just that, if there is one, it is not due to some kind of intrinsic temporal order, an order due to the nature of time itself. Instead, it is an order due to some other physical property, be it a temporal property or otherwise. The only necessary temporal aspect to this is it holds between events at different times.

'Anisotropy' refers to properties of something which are different depending on direction. Change the direction, and you change such anisotropic properties. What is allowed in Pricean physics is that the direction in time is determined by something which is not intrinsically a matter of temporal direction. For example:

[S] uppose time is finite in one direction, infinite in the other; or more granular in one direction than the other (in the sense that the gaps in discrete time get progressively smaller). In both cases, it would seem reasonable to say that time itself was anisotropic. (Price 2013, 292)

Although this is a global and absolute ordering of a continuous series of times, it is ordering in terms of two temporal properties which are not necessarily directional: distance from a temporal limit (the finite end of all of time) and the relative size of temporal gaps. For example, a period of time could be finite at both ends (such as a Gold universe in Price 1997) so that, from either direction, there is an increase in distance from a temporal limit.

In addition, Price even allows for a local order to time. What is not allowed by Pricean physics is a global or absolute order of time:

[A] precondition of any relevant notion of the direction of time is that it be possible to assign a temporal direction at every place and time, in a consistent way. In other words, we want to be able to pick a temporal direction, and label it (say) the positive direction, without our labels suddenly changing, as we move from place to place. (Price 2013, page 285) 
That is, for temporal order, the order of events are the same no matter the variation otherwise between them - in terms of other temporal properties (such as distance or scale) or other physical properties (such as entropy).

As such, if there is something to psychological time which distinguishes it from physical time (at least, according to Price), it is a global and absolute temporal order. It is not just local temporal order or temporal anisotropy defined by non-temporal properties.

Riemer does not distinguish such a global and absolute order from other kinds of anisotropy in time. He might assume it but it isn't clear anything that follows in the paper reveals or requires such an assumption. Further, to assume it is to commit one to a much stronger position than is necessary for psychophysical research. To show such global and absolute order in psychological time, one would need to show that psychological time has an order which is shared by all events, for all observers, all points of view, and all frames of reference.

\subsection{Holding That Physical Anisotropy is the Same as Psychological Anisotropy}

Finally for this section, given the previous discussions, one can have two ways that physical and psychological anisotropy are reversible, or not, in the same way.

First, it is consistent with Pricean physics that what seems to be temporal anisotropy in physics is actually events at different times ordered due to some non-temporal physical property. The most discussed example has already been mentioned -- entropy or disorder (a common analysis in physics, e.g., Horn 2013). If one event at one time is more disordered than another event at another time, the order due to increased entropy is a kind of temporal anisotropy.

This opens a way that psychological time may be consistent with physical time. The order in psychological time may be due to non-temporal physical differences. The difference between memory, perception and anticipation is often given by theorists of time perception to be an example of our psychological direction in time (e.g., Mellor 1998). If the differences between memory, perception and anticipation are explicable differences in physical structures, then there is no need to posit an additional psychological temporal order. For example, it might be due to differences in physical structures due to differences in the entropy of those structures (Bardon 2013).

Second, one threat to a Pricean concept of physical time may come from other physical theories. For example, thermodynamics is a physical theory (even if a special or emergent physical theory). Described a certain way, thermodynamics seems to require global temporal order if one holds it to be exhaustively described as follows: the second law of thermodynamics states that there is an increase in entropy in the universe. This 'increase' implies an order: reverse the order in time and it becomes a 'decrease'. If this second law holds globally and absolutely in time, and the increase cannot be explained in terms of other physical phenomena, then it implies a global and absolute order in time (see Thorn 2013 for discussion). If this is right, then the physical direction of time is irreversible -- meaning that, if the psychological direction is irreversible, there is no difference between them.

Riemer touches on this last point in referring to the Loschmidt Paradox [Riemer, page 2 of review draft]. This paradox is a paradox in physical time itself -- concerning how time- 
symmetric processes, such as microphysical phenomena, can give rise to time-asymmetric physics processes, such as those defined under the second law of thermodynamics.

To conclude this section, regarding the relationship between physical and psychological time, this paper raises concerns about two concepts:

a. Temporal anisotropy, which is due to non-temporal differences between events at different times and can be local. This is not a problem for physics, even Pricean physics (which denies the stronger temporal order). As such, it is not a problem regarding the relationship between the psychological and the physical.

b. Temporal order, which is global and irreducible to non-temporal differences between events at different times. This is a problem for Pricean physics. However, it is not a problem for all physical theories and is within physics itself, not between physics and psychology. Further, as a stronger kind of order - with the stronger ontological commitment of an absolute and global order -- more has to be done to show that it is a correct description of psychological anisotropy.

\section{Empirical Research and Anisotropy}

The main section of Riemer's paper is an examination of alleged unique problems that temporal anisotropy has for a number of methods in empirical research. Riemer's examples are examples of limitations and errors for time perception. And they do, indeed, seem to be limitations from time. However, the claim of this paper is there is something more here; these limitations are special. Other kinds of perception do not have equivalent limitations and errors.

Yet, before holding that temporal anisotropy has unique problems for psychophysics and empirical investigation, it needs to be shown that there are no analogues to these problems regarding other properties. If there are analogues, then the issues are not unique to time.

The most notable analogues of time and temporal anisotropy are space and spatial anisotropy. As will be discussed here, the examples Riemer gives seem to have spatial counterparts. The only difference with the examples and their spatial counterparts are that, with time, the stimuli are separated by times whereas, with space, the stimuli are separated by space.

To show this similarity, for each example problem, we change the temporal references to spatial references. ${ }^{2}$ If this results in plausible spatial problems, then the challenges are not uniquely temporal. And, if they are problems with anisotropy, they apply to spatial anisotropy as well.

\footnotetext{
2 This approach mimics the approach by the philosopher Schlesinger in his examination of what he calls the Doctrine of the Similarity of Space and Time (Schlesinger 1980, p.6). As its name suggests, this is the doctrine that time and space resemble each other.

Schlesinger is ultimately sceptical of this similarity for many fundamental temporal features. However, initially, he argues that some features of time have spatial analogues, and vice versa. Further, these analogues need not be obvious. For example, Schlesinger argues, contrary to a claim by Goodman 1964, just as with space, things can approach and recede from one another in time (Schlesinger 1980, 4-6).
} 


\subsection{Judgements of Earlier and Later Durations}

Similarly, Riemer gives the example of difference in judgements between earlier and later stages of a perceived duration, citing evidence which suggests that our judgements tend to be more erroneous in earlier trials than later trials. He argues that this is a particular complication with comparing durations, one not found in comparing other properties (such as weight or length). [Riemer, page 3 of review draft]

This is an interesting feature to account for in the reproduction of trials over time. When we reproduce trials for other properties, such as length, at different times, we may get different results for each trial which has nothing to do with the trials themselves but is only due to this earlier/later accuracy. Still, by knowing the earlier/later discrepancy, we can account for it.

Yet, as Riemer argues, it looks as if this may limit trials for temporal judgements. We cannot, at an instant, produce a duration. A duration just is a temporal extent. If that extent is anisotropic, then it has earlier and later stages. As such, one might argue, there will be this difference in accuracy for every trial. We cannot distinguish it between trials, and so cannot eliminate it for some of them.

However, this need not be a unique limitation for trials about temporal perception over time. It can also be found with trials for perception of other extents, such as spatial perception. The difference is that the trials are over the other extents, such as over space, instead of time.

Consider the following example: a subject sees a bowl of otherwise identical fruit. The bowl is so large that only some of the fruit occupy the centre of the subject's visual field.

In the subject's visual field, there is spatial anisotropy: there is a an ordering of detail from the visual centre to the edge (a decrease in detail) which is spatially irreversible; change the direction in space - from the edge to the centre - and the variation in detail is different (the detail increases). Further, this difference may alter our trial success regarding the less detailed objects on the visual edge: it does so if, because we have access to the central fruit/pattern, we make different (and in this case more accurate) results about the fruit at the edge.

If this is right, then the spatially anisotropic difference creates a difference in our judgements about the edge of our visual field. This is similar to a temporally anisotropic difference (earlier/later) creating a difference in our judgements about the later part of a duration.

\subsection{Reproduction}

Riemer describes a possible subject who suffers from having no short-term memory [Riemer, page 12 of review draft]. He cannot remember the standard duration while he has no problem with reproduction. The problem is that we cannot distinguish this from his inability to reproduce a stimulus.

He only has short-term memory of seven seconds. He is asked to reproduce two durations: three seconds and five seconds. He can reproduce three seconds because he can remember the three seconds and then accurately reproduce it within the space of seven seconds. But he cannot reproduce the five seconds. Although he might remember the five seconds within his window of seven seconds, the remaining time (of two seconds) is not enough for the five seconds needed for reproduction.

If the subject could remember what he is being asked to reproduce, he could reproduce it. However, he cannot remember it. Thus, he cannot reproduce it. But his inability to reproduce 
is only due to his window of seven seconds. It has nothing to do with a specific inability to reproduce.

There is an analogous problem with space and reproduction. Simultagnosia is a condition (found, for example, in Balint's Syndrome (Robertson 2004)) in which a subject cannot see multiple objects at one time. Given a number of objects in the space before them, they can only observe one at a time. As Riemer imagines a possible subject with no short term memory, let us imagine a possible subject of simultagnosia. The subject who can only observe 45 degrees $^{3}$ of their visual field at one time. If asked to reproduce 20 spatial degrees, they can do so, because observation and reproduction (totaling 40 degrees) can be encompassed by 45 degrees. But they cannot reproduce 30 degrees; the observation and reproduction of 30 degrees totals 60 degrees which is beyond their spatial limit of 45 degrees.

One might object that, to reproduce, one needs time. One needs to observe something and then, after observing it, reproduce it. To have reproduction, one needs both the original trial and the reproduction trial.

In response, given it concerns observations at a time, one also needs a different space for reproduction: one needs to observe something and then, somewhere else, reproduce it. The observed space is occupied by something at that time.

It's not obvious, once we have distinguished that the different kinds of problems raised here are about time and space, that there is much difference between them.

\subsection{Time-Perception and Memory}

To highlight a particular time-related failing, Riemer presents models of temporal experience, in particular of perceived change, as involving memory [page 9 of review draft]. Further, he states, time perception is particularly mediated or indirect. As an example of this view, he gives the views of an influential phenomenologist of temporal experience, Edmund Husserl. He then argues that this necessity of memory for time perception raises special errors not found in other kinds of perception.

First, note that Husserl's view of time perception varied over his lifetime and the account generally ascribed to him by later theorists does not involve memory as such (e.g., Kelly 2005). It involves retentions and protentions. The view that memory is appropriate for time perception is more the view of Plumer (Plumer 1984) or, less explicitly, Mellor (Mellor 1998).

In addition, the Husserlian view is not the only influential contemporary view of time perception. There is also the view of the philosopher of temporal experience, Dainton. Dainton does not consider temporal experience to seem mediated or indirect. And, unlike Husserl, Dainton explicitly considers contemporary physical theories of time in his work (e.g., Dainton 2000). If one is to take physics seriously in psychological work, one should not assume any of Husserl's view are correct (they may be, of course).

\footnotetext{
${ }^{3}$ The actual examples of simultagnosia seem to be tied to objects rather than numerically measured parts of a visual field. Riemer's example uses short-term memory that has numerical values of clock time. Yet, it is unlikely that whatever constraints are on a subject's temporal window is determined by a clock value. Clock time is a conventional and social metric. Without argument, one should not assume that a subject's experience is determined by a conventional or social metric (rather than something else, for example, physiological, internal, or subjective).
}

Commented [SH3]: Maybe this part could be cut entirely, it doesn't seem central to me.

Commented [PSE4R3]: Assuming that philosophers working on time consciousness will be interested in this piece, and Riemer's piece, I'm keeping this section in. But even if they did not, I think it is important - as a philosophical response - to highlight how Riemer's gloss on temporal experience does not match contemporary thinking about temporal experience (amongst philosophers and phenomenologists, anyhow).

Riemer presents a description of temporal experience that many contemporary theorists about time perception would reject in one way or another (e.g., it doesn't involve memory, or it isn't mediated or indirect - or doesn't seem to be, anyhow).

Also, those who do resist the sort of view glossed here by Riemer include philosophers cognisant of the physical concept of time, which is relevant to this specific paper. 
Second, memory may be involved but why is that an issue which makes time perception unique? The concept of memory can be very complex. However, it seems to be a minimal and most general condition of it that it be a cognition (for example, a belief or experience) about the past. An error of memory is an erroneous cognition about the past. This is obviously temporal.

But there are equivalent spatial errors. One can have cognitions of other places, such as what is behind you, with such erroneous cognitions about those other places. And this is obviously spatial.

\subsection{Direct Phenomenal Existence}

The final issue concerns the claim that, unlike other properties, temporal properties such as duration and order cannot have what Riemer calls 'direct phenomenal existence'. I think this claim reveals the real difference between time and space in the discussion. It is a difference in ontological assumptions, in the metaphysics of time and the metaphysics of space. Regarding the difference between the psychological and physical, it falls amongst the physical. However, although it is an intuitive assumption, it is also not an assumption of the majority of contemporary philosophers of time. Indeed, where it is understood, it is explicitly rejected by philosophers who accept the kind of physical view of time expressed in this paper.

Riemer states that time perception is distinguishable from other properties because it does not involve a 'phenomenally existent quality':

Beyond the short time window sometimes referred to as sensible present or specious present, which is assumed to be less than three seconds (Pöppel, 2009; Wackermann, 2007), a duration is phenomenally not existent in the same way as the length of a line or an object's weight.

[Riemer, page 6 of review draft]

As accepted here, the direct phenomenal existence of some duration -- of the specious present -- is analogous to the direct phenomenal existence of length or weight. As such, there is no difference between duration and other properties.

However, Riemer goes on to discuss other kinds of temporal experience which, he claims, are not existent like this. Once we go beyond the specious present, we move to experiences of much larger durations - minutes, hours, days. I agree that we do not perceive these durations the way we perceive directly perceived lines and weights. However, this is not a fair comparison. Not all durations have direct phenomenal existence but neither do all lines and weights have direct phenomenal existence.

If I stand at a subway platform, I can directly perceive some of the subway track but not all of it. I cannot perceive the track receding out of view and I cannot perceive the line between two contiguous atoms within the steel rails. Many lengths of the track lack direct phenomenal existence. Similarly, the weight of a bag of oranges in my hand may have direct phenomenal existence but the weight of the Earth's atmosphere, or a mountain falling on me, or a bacterium on my skin - none of these have direct phenomenal existence. In general, neither the shape, weight nor age of any particular atom or the universe as a whole has direct phenomenal existence.

Here is what the durations without direct phenomenal existence seem to have in common: They are too small (e.g., the milliseconds of a moving bullet) or too large (e.g., the 
eons of evolution). Yet, some lines and weight also have that in common and similarly lack direct phenomenal existence: they are too small (e.g., spaces between atoms; bacterial weight) or too large (e.g., the universe's length and weight).

In order to show that there is a difference here between space, time and weight, one must show that the lack of direct phenomenal existence for some durations is unique; there is no equivalent lack for lines and weights. Still, that difference is not found in this account.

\section{The Relevance of Philosophy and Physics to Psychophysics}

One reason to think duration lacks direct phenomenal existence is because one thinks that only what is at one time can have direct phenomenal existence. If this is right, then it is indeed correct to deny that duration can have such an existence. If what is only at a single point in space could have direct phenomenal existence, then what has length could not.

However, why assume that only what is at one time has such existence? Examples of the specious present indicate that it is not because that is how it seems: the specious present extends over a period of time, and so is not at a single point in time.

One reason may be that one assumes a particular intuitive $e^{4}$ position on the nature of time. I want to suggest here that this position, whether explicit or implicit, may contribute to the assumption that time is special in some way. But I also want to suggest that this intuitive position is not the one that should be taken if one is committed to contemporary physical concepts of time.

\subsection{The Philosophy of Time}

Many philosophers consider an intuitive position on time to be presentism. Presentism is the view that only what is at one time - the present - is real or exists (e.g., Bigelow 1996, Crisp 2005). If presentism is true, then, if something must have existence to have Riemer's direct phenomenal existence, anything with direct phenomenal existence must be at one time. By definition, duration is unable to be at one time. Thus, given presentism, duration cannot have direct phenomenal existence (or any other kind of existence, for that matter).

\footnotetext{
${ }^{4}$ One might worry what is meant by an 'intuitive' concept or position. It might be thought that 'intuitive' refers merely to introspection (or some process similar to those accepted by mathematical intuitionists) or something merely subjective. However, this is not the case.

In contemporary philosophical debate, 'intuitive' is often used to mean the 'folk' or pre-reflective concept one has of something. It is what many call a 'folk' concept.

As such, some defenders of presentism (also known as presentists) might be claiming that presentism is 'intuitive' in the sense that this is most people naturally or pre-reflectively think. If that's what is meant, a presentist is defending a folk concept, with all the commitments that might entail (which, as with introspection, may be no commitments at all).

However, many presentists claim something stronger -- that, whether we know it or not, presentism is the position which everyone holds about time. It does not matter whether one is a theorist about time or a member of the 'folk'. One reason given by such defenders is that the core belief of presentism - that only the present is real -- is a 'common sense' belief (adapted from Moore 1925), a belief which everyone holds (Moore's example is the belief in an external world). For an explicitly 'Moorean' defense of presentism, see Crisp 2005. For similar arguments, see Bigelow 1996 and Geach 1998.
} 
However, even if presentism is intuitive, this is a philosophical position that is rejected by many (if not most) philosophers of time. Considering the subject of this paper, philosophers' reasons for rejecting presentism are also reasons for psychophysicists to reject the position.

Presentism is incompatible with the physical theory of relativity (e.g., Mellor 1998). Relativity theory is part of the physical theory which motivates Price's position that time lacks temporal order. As such, if one considers Price's views on time in one's psychophysical theory, one is considering a theory which is incompatible with presentism.

It is also worth noting that, if the specious present has direct phenomenal existence, then it is a duration that has direct phenomenal existence. As such, given direct phenomenal existence requires existence ${ }^{5}$, it is incompatible with presentism (see Power 2012 and Power 2015).

If this is right, then presentism is a philosophical position which is incompatible with contemporary psychology and contemporary physics. Those who hold this position have problems which are not unique challenges merely from time for psychophysical research. The problems are challenges from their philosophical positions about time. Alternative positions on time might not have these problems.

\subsection{Relativity Theory}

The foregoing discussion may raise a lingering worry about its relevance to psychophysics. This concerns relativity theory.

The worry is that the physical theory of relativity is irrelevant to psychophysical research. Given the relative speeds at which the subjects of any such research move relative to each other, empirically observable effects predicted by relativity theory are undetectable. As such, the differences in the temporal and spatial measurements of an event due to different subject's motion are undetectable in psychophysical research.

However, this does not make relativity theory itself irrelevant. Relativity theory also defines any ontology which is relevant to models and theorising. The theory makes claims about the structure of space and time. For example, given relativity, there is no non-arbitrary simultaneity between events (or even any real simultaneity at all; see Maudlin 2012). As such, if one holds to relativity theory, one ought not to assume simultaneity in one's models and theories. In this way, relativity theory is relevant to current psychophysical research.

Indeed, in this way, presentism can also be relevant. Presentism holds that only present things are real, and all present things are simultaneous. As such, unlike relativity, one ought to assume simultaneity between the things in one's models and theories.

However, unlike presentism, relativity is the most accepted theory of time in physics. For psychophysics, this means that any models or theories one has about how the psychological and the physical relate to one another should be compatible with relativity theory but not presentism (for discussion on how relativity might affect the choice of models of timeconsciousness, see Power 2010).

In conclusion, Reimer's overall claim may be right - that there are unique challenges from time and temporal anisotropy for psychophysics. However, to ensure that, one should examine those challenges in light of theories of time which are physical theories (such as

${ }^{5}$ One could argue that direct phenomenal existence does not need existence. In that case, there is even less reason to deny that duration has it. 
relativity theory) and be careful that one is not assuming theories which, intuitive as they may be, are not (such as presentism).

\section{References}

Bardon, A. 2013. A Brief History of the Philosophy of Time. Oxford, Oxford University Press Bigelow, J.1996. "Presentism and Properties." In Noûs, 30 (10), 35-52

Crisp, T. 2005. Presentism and "Cross-Time" Relations. American Philosophical Quarterly, 42(1), 5-17

Dainton, B. 2000. Stream of Consciousness: Unity and Continuity in Conscious Experience. London, Routledge

Dainton, B. 2001. Time and Space. Chesham, Acumen

Geach, P. 1998. Some Problems with Time. In Metaphysics: The Big Questions. London, Routledge, eds. Van Inwagen, P. \& Zimmerman, D., 192-203

Husserl, E. 1964. The Phenomenology of Internal Time-Consciousness. The Hague, Indiana University Press

Kelly, S. D. 2005. The Puzzle of Temporal Experience. In Cognition and the Brain: The Philosophy and Neuroscience Movement. Cambridge, Cambridge University Press, ed. Brook, A \& Akins, $K$.

Moore. G.E. 1925. A Defense of Common Sense. In Contemporary British Philosophy (second series). London, Allen \& Unwin, 192-233, ed. Muirhead, J.H.

North, J. 2013. Time in Thermodynamics. In The Oxford Handbook of the Philosophy of Time. Oxford, Oxford University Press, ed. Callender, C.

Plumer, G. 1984. The Myth of the Specious Present. Mind, 94 (373), 19-35

Power, S.E .2010. Complex Experience, Relativity and Abandoning Simultaneity. Journal of Consciousness Studies, 17(3-4), 231-256

Power, S.E. 2012. The Metaphysics of the 'Specious Present'. Erkenntnis, 77 (1), 121-132 Power, S.E. 2015. Relative and Absolute Presence. In Philosophy and Psychology of Time (Studies in Brain and Mind). (Forthcoming), Springer

Price, H. 1997. Time's Arrow and Archimedes Point: New Directions for the Physics of Time. Oxford, Oxford University Press

Price, H. 2013. The Flow of Time. In The Oxford Handbook of the Philosophy of Time. Oxford, Oxford University Press, ed. Callender, C.

Robertson, L. C. 2004. Space, Objects, Minds, and Brains. Sussex, Psychology Press

Schlesinger, G. 1980. Aspects of Time. Indianapolis, Hackett Publishing Company 